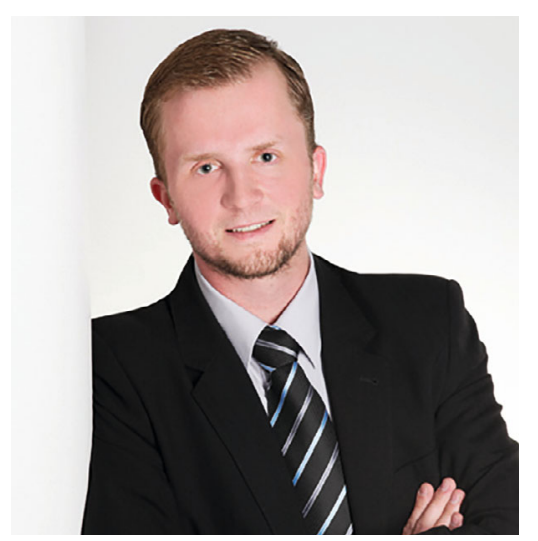

DR. SEBASTIAN FELD

Dr. Sebastian Feld ist Leiter des Quantum Applications and Research Lab (QARLab) am Lehrstuhl für Mobile und Verteilte Systeme der LMU München. Derzeit verfolgt er das Ziel der Habilitation mit dem Fokus auf Optimierungsproblemen und der Anwendung von Quantentechnologien. Sebastian Feld kam 2013 an die LMU und promovierte 2018 in den Themenbereichen Alternativrouten, Zeitreihenanalyse und geospatiale Trajektorien. Zuvor war er wissenschaftlicher Mitarbeiter am Institut für Internet-Sicherheit in Gelsenkirchen und arbeitete in den Themenfeldern Internetvermessung, Identitätsmanagement sowie Penetrationstests. Seit seiner Zeit im Freistaat Bayern hat Sebastian Feld mehrere Forschungsprojekte koordiniert, unter anderem das vom bayerischen Wirtschaftsministerium geförderte Projekt „Das Mobile Internet der Zukunft" sowie das an das Zentrum Digitalisierung Bayern (Z.DB) angegliederte Projekt „Innovationszentrum Mobiles Internet”.

\title{
Das Internet der Dinge - Von der Konnektivität zum Mehrwert
}

Die Geburtsstunde eines Buzzwords

Wann ein Begriff, der viele Jahre später von vielen Millionen Menschen ganz selbstverständlich im Alltag verwendet wird, tatsächlich zum ersten Mal in den Umlauf gebracht wurde, ist oft schwer zu sagen. Noch schwerer scheint es manchmal, den Begriffsbestimmer selbst zu identifizieren. Im Falle des Begriffes „Internet der Dinge“ verhält es sich möglicherweise anders. Kevin Ashton räumt zwar ein, dass er falsch liegen könnte. Ashton sei sich jedoch recht sicher, dass der Begriff „Internet der Dinge“ 1999 das erste Mal von ihm selbst verwendet wurde. In einer Präsentation über das Thema Supply-Chain-Management bei dem Konsumgüterhersteller Procter \& Gamble soll Ashton die seinerzeit brandheißen Themen RFID und Internet miteinander in Bezug gebracht haben. Die Idee hinter seinem Konzept ist damals wie heute so einfach wie genial: Bislang waren Computer - und somit auch das Internet - fast vollständig abhängig von Menschen und deren Informationen. Nun sollen Computer dazu befähigt werden, Informationen zu sammeln, sodass sie selbst die Welt sehen, hören und schmecken können.

\section{Die Vision der technologischen Allgegenwart}

Und ist Kevin Ashton der Vater des Begriffes ,Internet der Dinge“, so müsste Mark Weiser gewissermaßen der Großvater sein. Denn: 1991 hat Weiser in einem Aufsatz mit dem Namen „The Computer for the 21st Century“ den Begriff „Ubiquitous Computing“ geprägt, zu deutsch etwa „allgegenwärtiges Rechnen“. Die beschriebene technologische Allgegenwart, die diese Bezeichnung meint, ist im Grunde durch zwei parallel verlaufende Entwicklungen entstanden: zum einen die zunehmende Verfügbarkeit von Rechenleistung und zum anderen die abnehmende Sichtbarkeit der Technologien. Indirekt spürt jeder von uns täglich, dass die Informations- und Kommunikationsnetze allgegenwärtig geworden sind. Vor einigen Jahren nahm es noch mit der enormen Verbreitung und Nutzung von Mobiltelefonen seinen Anfang. Heute ist die Omnipräsenz nun in die Einbettung mobiler Transceiver in Gadgets und Alltagsgegenständen gemündet. Diese großartige Entwicklung hat zu einer neuen Dimension der Konnektivität geführt: Wir kommunizieren jederzeit (anytime) und überall (anyplace) mit allem (anything) und jedem (anyone). Diese zunehmenden Verbindungen führen zu einem neuen, dynamischen Netz aus Netzen: dem Internet der Dinge.

\section{Technologien für ein Internet der Dinge}

Das Internet der Dinge ist ein vornehmlich - wenn auch nicht ausschließlich - technisches Thema. Um die Vision der technologischen Allgegenwart Realität werden zu lassen, sind grundsätzlich vier Technologien von zentraler Bedeutung. Für jeden Anwendungsfall und für jede professionelle Domäne kann dies zwar unterschiedlich ausfallen. In ihrer Grundstruktur scheinen die vier Teile jedoch stets verwendet zu werden.

Erstens ermöglicht die Identifizierung von Objekten die Anbindung von Gegenständen und Geräten mit Datenbanken, Netzen und dem Internet und somit die Erfassung und Verarbeitung von Daten über diese Dinge. Moderne Car Sharing-Angebote wären ohne eine Identifizierung nicht möglich (das Auto muss im System identifiziert werden und der Kunde muss seine Identität nicht nur dem Anbieter, sondern auch gegenüber dem Auto beweisen) oder auch mobiles Bezahlen (Kunde identifiziert sich am Smartphone, das Smartphone an der Supermarktkasse, ...), um nur zwei Beispiele zu nennen. Die Identifizierung von Objekten soll dabei möglichst einfach, unauffällig und kosteneffektiv sein. Eine weit verbreitete Technik ist beispielsweise die Radio-Frequency Identification (RFID).

Zweitens werden mittels Datenerfassung Statusänderungen von Dingen automatisch erkannt. Sensortechnologien leisten also eine enorm wichtige Arbeit; sie verbinden die physische mit der virtuellen Welt. Auch Datenerfassung ist für viele moderne Dienste unverzichtbar, denn ohne Sensoren und Aktoren wäre ein Smart Home nicht smart und ein autonomes Auto nicht autonom. 
Drittens wurde im Zuge des Internets der Dinge der Begriff der eingebetteten Intelligenz immer wichtiger. Dieser besagt, dass die Fähigkeit zur Informationsverarbeitung aus dem „Zentrum“ des Internets an den „Rand“ der Netze verlegt wird. Mit anderen Worten: Wenn früher nicht-smarte Geräte Informationen gesammelt, damit Anfragen an Server gesendet und auf die entsprechenden Antworten gehorsam reagiert haben, so soll dieser „Umweg“ nun obsolet werden. Statische Objekte werden zu dynamischen Dingen, die unabhängig Entscheidungen treffen können. Dies erhöht - ganz nebenbei - die Leistungsfähigkeit und die Widerstandsfähigkeit des Netzes selbst. Beispiele für eingebettete Intelligenz finden sich an vielen Stellen. Es fängt an bei Radioweckern, die die Helligkeit des Displays automatisch an die Umgebungshelligkeit anpassen, und hört auf bei mobilen Robotern, die tatsächlich autonom, also ohne fremde Einwirkung, agieren.

Schließlich ist die Miniaturisierung und Nanotechnologie ein essenzieller Bestandteil des Internets der Dinge. Immer kleinere Dinge können interagieren und sich verbinden, was im Alltag mindestens durch Notebooks, Tablets, Smartphones und Smartwatches ersichtlich ist. Diese Entwicklung geht aber natürlich noch weiter, viele Beispiele finden sich etwa in der Medizin. Derzeit wird an smarter, kontinuierlicher Blutzuckermessung geforscht, an automatisierter Insulinzufuhr oder an einnehmbaren Sensoren, die für eine gewisse Zeit im menschlichen Körper verbleiben.

Zusammengefasst erschafft die Kombination der genannten Entwicklungen ein Internet der Dinge, das Objekte sensorisch und intelligent miteinander verbindet.

\section{Herausforderungen für einen nachhaltigen Erfolg}

Auch wenn der Teufel bekanntlich im Detail steckt, so sind wiederkehrend verschiedene grundlegende Herausforderungen zu meistern, um einen nachhaltigen Erfolg für das Internet der Dinge zu gewährleisten.

Eine der weitreichendsten Herausforderungen ist die technologische Standardisierung. Sie hat sich als wesentlich für die Verbreitung und den Masseneinsatz vieler Technologien erwiesen, und so wird es sich auch mit den unterschiedlichsten Realisierungen des Internets der Dinge verhalten. Das klassische Internet oder auch das moderne Mobilfunknetz wären ohne Standards wie TCP/ IP oder IMT-2000 nicht möglich. In zahlreichen Foren, Gremien und Allianzen, die oft ,hinter den Kulissen“ arbeiten, werden Protokolle harmonisiert und eine Kompatibilität zwischen bestehenden und auch zukünftigen Technologien erwirkt.

Eine weitere grundlegende Herausforderung ist das Konzept von Datenanfrage und Dateneinwilligung. Dieses wird nämlich schnell obsolet, wenn Dinge mit „Sinnen“ sowie Rechen- und Kommunikationsfähigkeiten ausgestattet werden. Während in der physischen Welt ein Arzt Patientendaten erst an Dritte übersenden darf, wenn der Patient ihn ausdrücklich von der Schweigepflicht entbunden und der Übermittlung ermächtigt hat, so scheint in der virtuellen Welt oft ein kontinuierlicher Datenaustausch zwischen Dingen und Menschen sowie Dingen und Dingen unsichtbar für Eigentümer und Urheber stattzufinden. Hier müssen geeignete Wege gefunden werden, die sich nicht nur auf die Sicherheit beziehen, sondern auch auf die Benutzbarkeit, Verständlichkeit und Nachvollziehbarkeit.

\section{Geschäftsrollen im Ökosystem des Internets der Dinge}

Was das Internet der Dinge neben der Vielzahl an Ideen, Komfort und $\mathrm{Spa} ß$ außerdem höchst interessant macht, ist die Tatsache, dass das verwendete Ökosystem aus einer Vielzahl von Geschäftspartnern besteht, die alle mindestens eine Geschäftsrolle einnehmen. Die grundlegenden Geschäftsrollen, die im Internet der Dinge zu finden sind, lauten Geräteanbieter, Netzanbieter, Plattformanbieter, Anwendungsanbieter sowie Anwendungskunde.

Der Geräteanbieter ist verantwortlich für die Geräte, die Rohdaten und Inhalte für Netzbetreiber und Anwendungsanbieter gemäß deren Dienstlogik bereitstellen. Beispiele für solche Partner sind rein technische Hersteller, wie etwa Produzenten von vernetzbaren Wetterstationen.

Der Netzanbieter spielt eine zentrale Rolle im Ökosystem des Internets der Dinge und bietet den Zugriff und die Integration von Ressourcen anderer Anbieter an, die Unterstützung und Kontrolle von verwendeter Infrastruktur sowie das Angebot von Netzwerkfähigkeiten für andere Anbieter. Ein klassisches Beispiel für einen Netzanbieter, ohne den das Internet der Dinge nicht funktionieren würde, ist der lokale Internetanbieter wie etwa die Telekom.

Der Plattformanbieter stellt Integrationsmöglichkeiten und offene Schnittstellen zur Verfügung, mit denen beispielsweise Datenspeicherung, Datenverarbeitung oder Gerätemanagement durchgeführt werden können. Enorm präsente Plattformanbieter sind etwa Amazon mit AWS IoT oder Google mit Cloud IoT.

Schließlich verwendet der Anwendungsanbieter die Funktionen und Ressourcen der bereits genannten Partner, um neuartige Internet der Dinge-Anwendungen für Endnutzer bereitzustellen. Eine komplexe Anwendung wie ein Smart Home verwendet entsprechend Geräte, Netze und Plattformen.

Um nun aus der Konnektivität tatsächlich einen Mehrwert entstehen zu lassen, benötigt es schlussendlich den Anwendungskunden. Dieser kann sich natürlich im privaten als auch im geschäftlichen Umfeld befinden und verwendet die Anwendungen, die von den Anwendungsanbietern bereitgestellt werden.

\section{Von der Konnektivität zum Mehrwert}

Zusammengefasst ist das Internet der Dinge eine beträchtliche Entwicklung, die in den letzten zwanzig Jahren immer mehr von einer Vision zur Realität wurde. In vielen kleinen Schritten wurden Ideen ausprobiert, verworfen oder weiterentwickelt. Die Brisanz des Themas nimmt nicht ab, sondern immer weiter zu und die Bereiche der physischen Welt, in die das Internet der Dinge Einzug hält, werden immer zahlreicher und bunter.

Ich persönlich bin gespannt auf all die Entwicklungen, die in naher und ferner Zukunft Realität werden, und wünsche Ihnen viel Spaß bei der Lektüre dieser Zeitschrift. 